\title{
EFFECT OF A LIQUID MULTIVITAMIN/MINERAL SUPPLEMENT ON ANAEROBIC EXERCISE PERFORMANCE
}

\author{
Andrew C. Fry \\ Richard J. Bloomer \\ Michael J. Falvo \\ Chris A. Moore \\ Brian K. Schilling \\ Lawrence W. Weiss
}

Human Performance Laboratories, The University of
Memphis, Memphis, Tennessee, USA

The purpose of this study was to determine if supplementation with a liquid multi-vitamin/mineral would improve anaerobic exercise performance. Fourteen resistance-trained men performed a 30-second cycle sprint and one set of squat exercise on 2 separate days before and following 8 weeks of supplementation with either a liquid multi-vitamin / mineral or a placebo. Heart rate, perceived exertion, blood lactate, peak and mean power, and rate of fatigue were determined for all tests. No differences were noted for any variable $(\mathrm{P}>0.05)$. When controlling for presupplementation values, however, a decreased rate of fatigue was noted for both exercise tests following the multi-vitamin/mineral supplementation. These data suggest that in resistance trained men consuming a nutritionally sound diet, supplementation with a liquid multi-vitamin / mineral does not favorably impact most anaerobic exercise performances. Such supplementation, however, may result in a minor decreased rate of fatigue. It appears that, in terms of improved short duration anaerobic exercise performance, supplemental micronutrients may not be efficient ergogenic agents for well-trained individuals consuming an adequate diet.

Keywords: micronutrients, nutrition, ergogenic aid, dietary supplementation

Received 17 July 2005; accepted 11 November 2005.

Address correspondence to Andrew C. Fry, PhD, The University of Memphis, 135 Elma Roane Field House, Memphis, TN 38152, USA. E-mail: afry@memphis.edu 


\section{INTRODUCTION}

Vitamins make up one classification of micronutrients, functioning as organic catalysts in metabolic reactions within the human body. They are designated as nutrients because vitamins undergo continuous degradation, requiring exogenous replacement via dietary sources. Although vitamins are essential to support health and well-being, they are not direct sources of energy, and rather function to facilitate energy metabolism (Lukaski 2004). It has been suggested that adequate vitamin intake is of crucial importance for individuals engaged in strenuous physical exercise due to the increased metabolic activity during such work (Lukaski 2004).

A second class of micronutrients, minerals, assist in the formation of body tissues (e.g., calcium), maintenance of fluid balance (e.g., sodium and potassium), and excitation of tissues (Petrie, Stover, and Horswill 2004). In general, minerals are suggested to regulate macronutrient use via their biochemical functions, which also may have the potential to affect physical performance (Lukaski 2004). The combined effectiveness of these micronutrients is quite important, as they act synergistically with one another, influencing absorption and metabolism (Ash et al. 2003).

Although maintenance of the recommended values of micronutrients is well established for optimal health, the potential ergogenic effect of exogenous multi-vitamin/mineral intake has not been well supported (Singh, Moses, Deuster 1992; Telford et al. 1992; Weight, Myburgh, Noakes 1988). A possible exception to these findings includes the study of individuals with preexisting dietary deficiencies or ill health. In addition, the use of high dose isolated minerals has been reported to improve performance in some investigations (Brilla and Haley 1992, Krotkiewski et al. 1982; Richardson and Drake 1979).

In an attempt to enhance nutrient absorption, supplement manufacturers have developed multi-vitamin/minerals that are provided in liquid form. Such products have been suggested to be superior to typical vita$\mathrm{min} /$ mineral capsules or tablets, and have been touted as ergogenic aids. No study to date, however, has examined the efficacy of such supplementation in trained individuals who are consuming an adequate diet. Therefore, the purpose of this study was to determine if supplementation with a liquid multi-vitamin/mineral would improve anaerobic exercise performance in trained men who were consuming a nutritionally complete diet.

\section{METHODS}

\section{Subjects}

Fourteen, young (24 \pm 3 years) resistance-trained men were recruited to participate in this study. Following an explanation of all procedures, 
subjects completed a weight training and supplementation history questionnaire to verify their eligibility. Exclusion criteria included those who were not currently performing resistance training, incapable of performing a parallel back squat or stationary cycling, as well as those who were smoking or currently using vitamin/mineral supplementation or both. All subjects completed an informed consent document as approved by the Committee for the Use of Human Subjects for The University of Memphis and were considered healthy as indicated by questionnaire. Subject descriptive characteristics are presented in Table 1.

\section{Baseline Measurements and Familiarization}

Subjects were required to report to the Human Performance Laboratory on seven separate occasions. During their initial laboratory visit, at least one week prior to their first test, their height, weight, and percent body fat (as estimated via skinfold thickness) were measured and recorded. Following these measurements, subjects performed a one repetition maximum (1RM) test for the barbell squat on an instrumented Smith machine using methods previously described by Kraemer and Fry (1998). A Smith machine was chosen as it permitted the investigators to control the gross motor movement pattern for this exercise and allowed subjects to exert maximal force without concern about balance, as might be the case for the free bar squat. Following determination of the 1RM, researchers familiarized subjects with both anaerobic performance tests as described below (e.g., squat and cycle sprint tests). On separate occasions, subjects reported back to the lab to perform a second familiarization trial for both the squat and the sprint tests. Therefore, each subject had two familiarization trials for both the squat and the sprint test. This was done in order to allow for any potential "learning" effect with either of these tests. The additional four sessions were the actual experimental testing sessions.

Table 1. Descriptive Characteristics of 14 Resistance-Trained Men

\begin{tabular}{lcc}
\hline Variable & Multi-vitamin/mineral $(\boldsymbol{n}=\mathbf{8})$ & Placebo $(\boldsymbol{n}=\mathbf{6})$ \\
\hline Age $(\mathrm{yrs})$ & $25 \pm 4$ & $23 \pm 2$ \\
Height $(\mathrm{cm})$ & $176 \pm 7$ & $176 \pm 2$ \\
Weight $(\mathrm{kg})$ & $85 \pm 19$ & $90 \pm 14$ \\
Percent body fat & $10 \pm 3$ & $12 \pm 2$ \\
Squat 1-repetition maximum (kg) & $144 \pm 32$ & $165 \pm 48$ \\
Years weight training experience & $5 \pm 3$ & $4 \pm 2$ \\
\hline
\end{tabular}

Values are mean \pm SD. No statistically significant differences observed between treatment groups $(P>0.05)$. 


\section{Supplementation}

Subjects were randomized in a double-blind manner to receive either a micronutrient supplement $(n=8)$ or a placebo $(n=6)$. Subjects were instructed, in accordance with manufacturer recommendations, to ingest one ounce of the liquid supplement daily (1-Step Liquid MultiVitamin \& Mineral ${ }^{\circledR}$, High Performance Fitness; Hermosa Beach, CA). Each one ounce dosage provided the nutrient equivalents as reported in Table 2.

Supplementation began following the first test session (following the squat test as described below) and continued through the conclusion of

Table 2. Multivitamin/Mineral Supplement Description

\begin{tabular}{lrr}
\hline & \multicolumn{2}{c}{ Each 1 oz. serving contains the following: } \\
\cline { 2 - 3 } Ingredient & Amount & \% RDA \\
\hline Vitamin A/Beta Carotene & $7500 \mathrm{IU}$ & 150 \\
Vitamin B1 & $2.25 \mathrm{mg}$ & 150 \\
Vitamin B2 (riboflavin) & $2.55 \mathrm{mg}$ & 150 \\
Vitamin B3 (niacin) & $33 \mathrm{mg}$ & 150 \\
Vitamin B6 & $3.0 \mathrm{mg}$ & 150 \\
Vitamin B5/Pantothenic Acid & $15 \mathrm{mg}$ & 150 \\
Folic Acid & $600 \mathrm{mg}$ & 150 \\
Vitamin B12 & $9 \mu \mathrm{g}$ & 150 \\
Biotin & $450 \mathrm{mg}$ & 100 \\
Vitamin C & $90 \mathrm{mg}$ & 150 \\
Vitamin D & $600 \mathrm{IU}$ & 150 \\
Vitamin E & $45 \mathrm{IU}$ & 150 \\
Calcium & $60 \mathrm{mg}$ & 8 \\
Chromium & $150 \mu \mathrm{g}$ & - \\
Iodine & $150 \mu \mathrm{g}$ & 100 \\
Iron & $150 \mu \mathrm{g}$ & 66 \\
Magnesium & $100 \mathrm{mg}$ & - \\
Manganese & $5 \mathrm{mg}$ & - \\
Potassium & $100 \mathrm{mg}$ & - \\
Selenium & $150 \mu \mathrm{g}$ & - \\
Sodium & $5 \mathrm{mg}$ & 150 \\
Zinc & $23 \mathrm{mg}$ & \\
\hline
\end{tabular}

Each $1 \mathrm{oz}$. serving contains $<0.5 \mu \mathrm{g}$ of the following trace minerals: bismuth, boron, bromide, cerium, cesium, cobalt, copper, dysprosium, erbium, europium, fluoride, gadolinium, gallium, germanium, gold, hafnium, holmium, indium, iridium, kjeldhal, lanthanum, lithium, molybdenum, neodymium, nickel, niobium, nitrogen, platinum, praseodymium, rhenium, rhodium, rubidium, samarium, scandium, silicon, silver, strontium, tantalum, tellurium, terbium, thullium, tin, titanium, tungsten, vanadium, yiterbium, zirocnium.

Other ingredients: purified water, fructose, citric acid, natural cranberry flavor.

Percent daily values are based on a 2000 calorie diet. 
the second test session. Therefore, all subjects ingested either the micronutrient supplement or placebo for a duration of 8 weeks, after which time the postsupplementation sessions were performed. Subjects continued to use their assigned treatment until all post-testing was completed. Subjects were provided with a plastic bottle, capable of dispensing the correct daily dosage, which was refilled weekly by a research assistant who was not involved in the performance of the pre- or post-tests. Although we did not witness each subject ingest their assigned treatment, nor were subjects required to record the day and time of treatment ingestion, we did have regular (i.e., 2-3 days per week) interaction with subjects to discuss their treatment compliance and to reinforce the importance of the daily dosage schedule. Therefore, we assumed that the empty bottles returned weekly to our research assistant, coupled with subjects' acknowledgment of daily treatment intake, served as evidence of subject compliance. This assumption, of course, is a limitation of our study.

\section{Dietary Assessment}

All subjects were instructed to follow their normal diets during the supplementation and testing periods. Subjects completed a detailed, 6-day food record prior to their initial testing session, which included all food and drink consumed. The records were analyzed for total kilocalories, protein, carbohydrate, fat, vitamin $\mathrm{C}$, vitamin $\mathrm{E}$, and vitamin A intake (Diet Analysis Plus, ESHA Research; Salem, OR). These data are presented in Table 3. Results of the diet analysis were returned to subjects and they were instructed to maintain a similar diet throughout the duration of the study, which was reinforced regularly by the investigators.

Table 3. Mean Dietary Intake Assessed During a 6-day Period Prior to Beginning Supplementation

\begin{tabular}{lccccccc}
\hline Exercise test & Kcal & Protein & $\begin{array}{c}\text { Carbo } \\
\text { hydrate }\end{array}$ & Fat & Vitamin C & Vitamin E & Vitamin A \\
\hline $\begin{array}{l}\text { Multi-vitamin/ } \\
\text { mineral }\end{array}$ & $2755(733)$ & $154(78)$ & $295(80)$ & $110(34)$ & $55(32)$ & $7(4)$ & $962(537)$ \\
\begin{tabular}{l} 
Placebo \\
\hline
\end{tabular} & $3177(775)$ & $156(68)$ & $360(91)$ & $125(51)$ & $66(32)$ & $7(4)$ & $1015(401)$ \\
\hline
\end{tabular}

Note: Values are mean $\pm(\mathrm{SD})$. Macronutrients are presented in gram $(\mathrm{g})$ quantities. Vitamins $C$ and $E$ values are represented in milligrams $(\mathrm{mg})$; vitamin $A$ values are provided in retinol equivalents. No statistically significant differences observed between treatment groups $(P>0.05)$. 


\section{Testing Sessions}

Following an overnight fast, subjects reported to the laboratory 3 to 5 days following familiarization to perform the first sprint cycle test. Following a 10-minute rest period, subjects' heart rate was recorded using a Polar heart rate monitor. A five $\mathrm{mL}$ blood sample was then taken via vacutainer from an antecubital vein for determination of lactate concentrations. Immediately following the exercise tests as described below, heart rate and rating of perceived exertion (RPE) were noted and recorded. A second blood sample was then obtained.

In an attempt to avoid any potential muscle injury and soreness that may occur as a result of the squat test (and hence affect performance on the subsequent sprint test), the sprint cycle test was performed first. Subjects returned to the laboratory 3 days later to perform the squat test. At the completion of the squat test, subjects began their assigned treatment as described above. Both the sprint and the squat test were repeated following an 8-week supplementation period.

The sprint test consisted of a standard 30-second Wingate (WAnT) sprint cycle test, performed on a specially modified Monark cycle ergometer. This cycle is bolted to the floor and can be basket loaded with weight plates in order to apply tension to the flywheel. Additionally, the flywheel is interfaced with a switch connected to a printer, which records a mark on continuous running paper for each flywheel revolution performed. This allows us to calculate the total number of flywheel revolutions during WAnT with greater accuracy. Subjects maximally pedaled against a heavy load based on their body weight $\left(0.7 \mathrm{~N} \mathrm{~kg}^{-1}\right)$. Prior to this test, subjects lightly cycled on a stationary cycle interspersed with short (e.g., 5-second) sprints as a warmup. Peak and mean power, and percent fatigue (\% fatigue) were recorded.

The squat test was performed on a Smith machine at a load equivalent to $70 \%$ of their predetermined $1 \mathrm{RM}+$ body mass. Squats were performed in a full range of motion with mechanical stops positioned in the Smith machine to ensure proper depth (i.e., posterior thigh parallel with ground) and to maintain consistency between pre- and post-testing. Foot placement was recorded during determination of $1 \mathrm{RM}$ for replication in subsequent testing sessions through the use of foot placement grid. The test consisted of 15 repetitions at a cadence of one repetition per 6 seconds in accordance to a computerized metronome. Subjects were instructed to momentarily pause in the bottom position and then maximally accelerate through the concentric phase of the movement upon a verbal cue. Prior to the squat test, subjects performed free weight barbell squats for three sets of five repetitions, where one set was performed at a load equal to $30 \%$ $1 \mathrm{RM}$, and two sets were performed at a load equal to 50\% 1RM. Peak and mean power and percent fatigue were recorded. 


\section{Data Acquisition}

Peak and mean power and percent fatigue were determined from the WAnT cycle test by methods previously described (Kraemer et al. 2002). The following equations were used to calculate these variables:

Peak Power $\left(\mathrm{N} \cdot \mathrm{m} \cdot \mathrm{s}^{-1} ; \mathrm{W}\right)=\left[\mathrm{N} \times\left(\right.\right.$ revolutions $\left.\left._{\max } \times 6 \mathrm{~m}\right)\right] / 5 \mathrm{~s}$

Mean Power $\left(\mathrm{W} ; \mathbf{J} \cdot \mathbf{s}^{-1}\right)=$ total revolutions $/ 30 \mathrm{~s}$

$\%$ Fatigue $=[($ Peak Power - lowest power $) /$ Peak Power $] \times 100$

$(\mathrm{N}=$ Newtons, $\mathrm{W}=$ Watts, $\mathbf{J}=$ Joules $)$

Kinetic variables obtained during the squat test were acquired using a computer-interfaced dynamometer (Fitrodyne; Bratislava, Slovakia). Briefly, this device contains a spooled nylon tether that when attached to a barbell determines linear velocity via a velocity transducer. Through inverse dynamic calculations, the first derivative of velocity (acceleration) with respect to time is added to the acceleration due to gravity, the product of which is force. Calculated force is then multiplied by measured velocity to determine resultant power. These data are sampled at $100 \mathrm{~Hz}$ and linearly smoothed over a 50ms interval. Percent $(\%)$ fatigue was calculated using the same equation as used for the WAnT cycle test. In this equation, however, "peak power" was the single repetition with the highest power output, and was compared with the last repetition. This squat test has been reported to be reliable (ICC $\geq 0.811$ ) for this subject pool for all measures analyzed herein (Bidne et al. 2005).

\section{Statistical Analysis}

Dependent variables were analyzed using a 2-way (group $\times$ time) mixedmodel analysis of variance (ANOVA), or with independent $t$ tests. Additionally, presupplementation values were used as covariates to equate both groups for ANCOVA statistics for evaluating \% fatigue. Where appropriate, significant interactions and main effects were further analyzed using the Fisher LSD method. Significance was set a priori at $P<0.05$. All data are presented as mean \pm standard error of the mean (SEM), except anthropometric measures and dietary intake, which are presented as mean \pm standard deviation (SD).

\section{RESULTS}

All subjects completed testing sessions with $100 \%$ compliance. Subject characteristics were not significantly different between treatment groups 
(Table 1), nor were any of the measured dietary variables (Table 3 ). In relation to the dietary data, it appeared that subjects were consuming adequate kcals and macronutrients. In addition, all subjects had vitamin and mineral intakes that were, on average, at or above the recommended daily intake values. When collapsing treatment groups across both times, no difference was noted for total work performed between the WAnT and squat test ( $299.67 \pm 16$ vs. $314 \pm 16 \mathrm{~kJ}$, respectively).

Pre- and post-supplementation values for heart rate, lactate, and RPE are presented in Table 4. In addition, pre- and post-supplementation measures for peak power and mean power for both the WAnT and repeated squat tests are presented in Table 5. In relation to all of the above variables, no statistically significant differences were noted between the treatment groups $(P>0.05)$. When controlling for baseline values, however, we found that the percent rate of fatigue during both tests was less for the multi-vitamin/ mineral group compared with the placebo $(\mathrm{P}<0.05$, Figure 1$)$.

\section{DISCUSSION}

The present investigation determined the effect of 8 weeks of liquid multi-vitamin/mineral supplementation on measures of anaerobic exercise performance in resistance trained, well-nourished men. Specifically, we observed performance during both a 30 -second cycle sprint and one set of barbell squats in relation to peak and mean power, fatigue, and their associated responses (e.g., blood lactate, heart rate, RPE).

Results of this study demonstrated that 8 weeks of multi-vitamin/ mineral supplementation in resistance trained men does not, in general, provide an ergogenic benefit. No reductions were evident in mean lactate, heart rate, or RPE response when comparing pre- to post-supplementation for either treatment group. Likewise, no significant improvements in peak or mean power were observed for either the WAnT cycle or squat test. While we chose to use low volume anaerobic exercise bouts in the present study as has been done in previous work, it is possible that the inclusion of a higher volume of work (e.g., 5-6 repeated sets of squats and sprints) may have resulted in statistically detected differences between treatment groups. Likewise, considering our relatively small sample size, the use of a repeated measures crossover design with the inclusion of a wash-out period would have increased our statistical power, possibly increasing our ability to detect treatment differences. Future investigations may consider these factors in the research design.

Despite no treatment differences in the above variables, when percent fatigue was adjusted for presupplementation values in both performance tests, those in the multi-vitamin/mineral group experienced an attenuated rate of fatigue during both tests. Although it has been suggested that 


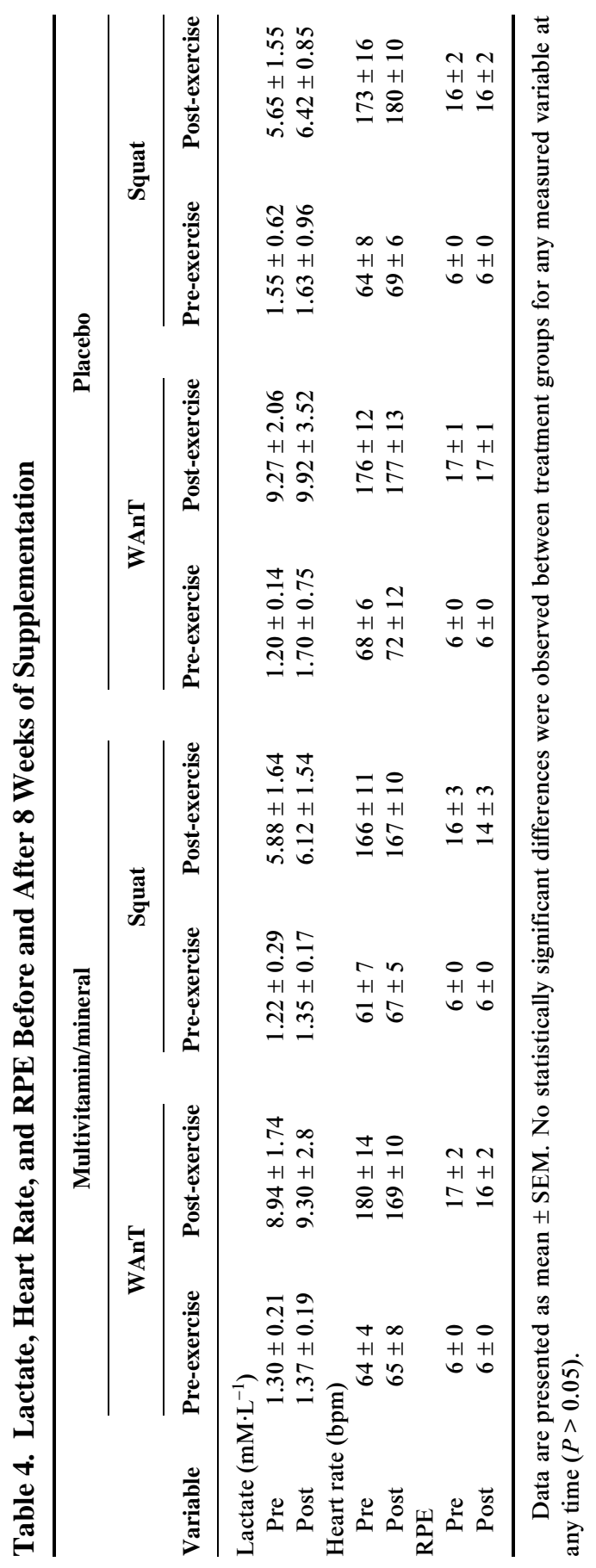


Table 5. Power Assessment Before and After Supplementation

\begin{tabular}{lccccc}
\hline & \multicolumn{2}{c}{ Multivitamin/mineral } & & \multicolumn{2}{c}{ Placebo } \\
\cline { 2 - 3 } \cline { 5 - 6 } Variable & WAnT & Squat & & WAnT & Squat \\
\hline $\begin{array}{l}\text { Peak power } \\
\quad \text { Pre }\end{array}$ & $447.95 \pm 139.47$ & $1320.36 \pm 288.35$ & & $483.17 \pm 99.28$ & $1428.83 \pm 292.63$ \\
$\quad \begin{array}{l}\text { Post } \\
\text { Mean power }\end{array}$ & $459.21 \pm 113.72$ & $1332.31 \pm 305.50$ & & $501.37 \pm 117.75$ & $1420.33 \pm 295.93$ \\
$\quad \begin{array}{lllll}\text { Pre } \\
\text { Post }\end{array}$ & $411.21 \pm 106.00$ & $1092.84 \pm 270.28$ & & $405.08 \pm 60.73$ & $1240.44 \pm 258.42$ \\
& $431.45 \pm 115.26$ & $1123.60 \pm 260.50$ & & $411.91 \pm 51.60$ & $1211.95 \pm 248.36$ \\
\hline
\end{tabular}

Data are presented as mean \pm SEM and are expressed in Watts $(W)$. No statistically significant differences were observed between treatment groups $(P>0.05)$.

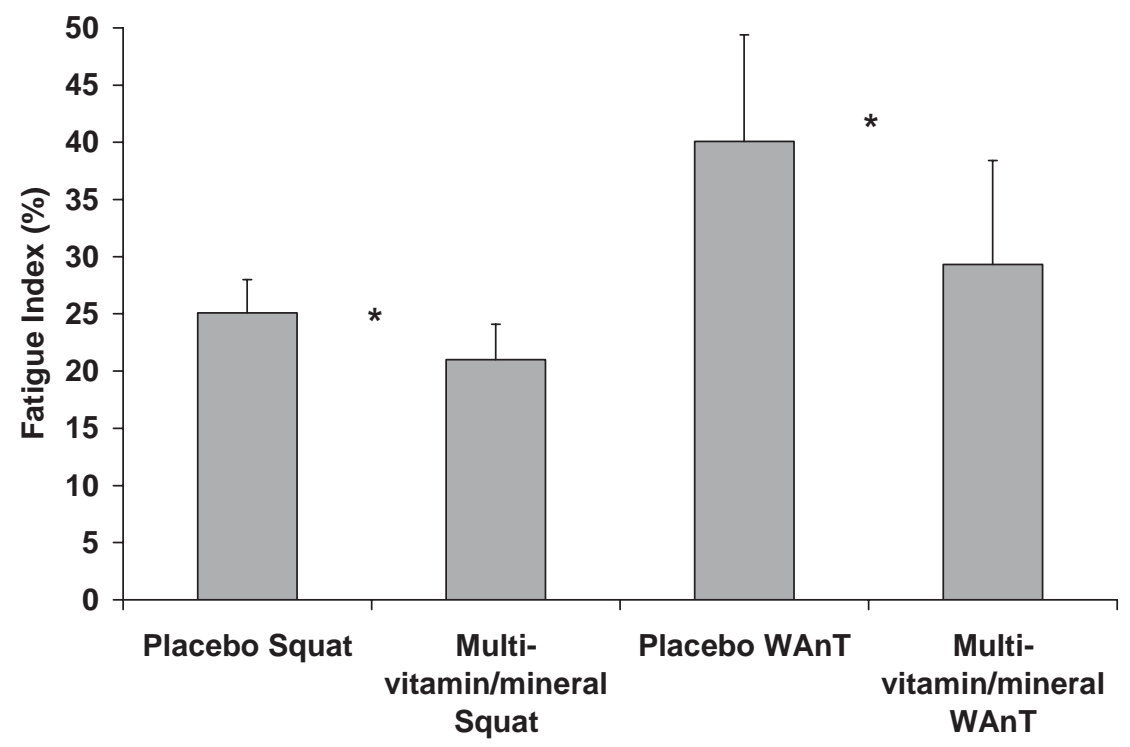

Figure 1. ANCOVA adjusted \% fatigue for the squat and Wingate Anaerobic (WAnT) tests for the placebo and multi-vitamin/mineral groups. $* P<0.05$.

micronutrient supplementation may have ergogenic effects (Lukaski 2004), the possible mechanisms of such an effect are beyond the scope of the present investigation.

Aside from a minor reduction in the rate of fatigue as suggested above, these data are in agreement with previous research that also failed to demonstrate an ergogenic effect of multi-vitamins and minerals. This appears 
true during both laboratory as well as sport-specific performance assessments (Singh et al. 1992; Telford et al. 1992; Weight et al. 1988).

Singh and colleagues (1992) were the first to provide a thorough description of the effects of a high potency multivitamin-mineral supplement on muscle strength and endurance. In this double-blind placebo controlled design, 22 healthy and recreationally active men performed a variety of performance measures (e.g., $\mathrm{VO}_{2}$ max test, submaximal running, and isokinetic knee extensions) prior to and following a 90-day supplementation period. Micronutrient supplementation did not have any clear effect for any physical performance parameter, nor did it have an effect on the blood lactate and heart rate response, which is in agreement with the current investigation.

While Singh et al. (1992) were the first to assess muscular strength and endurance following micronutrient supplementation, the performance measures were aerobic in nature (with the exception of knee extension exercise). The present study sought to study anaerobic performance through use of high power type exercise. We observed minor reductions in percent fatigue for both the WAnT cycle (placebo $=40.1 \pm 9.3 \%$; supplement $=29.3 \pm 9.1 \%$ ) and the squat test (placebo $=25.1 \pm 2.9 \%$; supplement $=21.0 \pm 3.1 \%$ ), with no differences observed between treatment groups for any other measured variables.

Taken together with the few prior reports on micronutrient supplementation and exercise performance, it appears that supplementation with a multivitamin/mineral in younger, healthy individuals who are well trained and well nourished provides only a modest ergogenic benefit. Therefore, supplementation with a mixed multi-vitamin/mineral product as used here in an effort to improve anaerobic exercise performance is not, in general, supported by the available evidence. These findings should be considered by sport nutritionists and others providing nutritional advice to healthy, active individuals interested in enhancing performance. While micronutrients are indeed an important nutrient class and certainly play an important role in an athlete's diet and supplementation regimen, they generally should not be classified as ergogenic agents for purposes of improved exercise performance.

\section{REFERENCES}

Ash DM, Tatala SR, Frongillo Jr EA, Ndossi GD, Latham MC (2003) Randomized efficacy trial of a micronutrient-fortified beverage in primary school children in Tanzania. American Journal of Clinical Nutrition 77: 891-898.

Bidne JS, Fry AC, Bloomer RJ, Schilling BK, Moore CA, Falvo MJ, Weiss LW (2005) [abstract] Reliability of the Memphis squat test. Las Vegas, NV: National Conference of the National Strength and Conditioning Association.

Brilla LR, Haley TF (1992) Effect of magnesium supplementation on strength training in humans. Journal of the American College of Nutrition 11: 326. 
Kraemer WJ, Fry AC (1995) Strength testing: Development and evaluation of methodology. PJ Maud, C Foster, eds. Physiological Assessment of Human Fitness. Champaign, IL: Human Kinetics 121: pp. 115-138.

Kraemer WJ, Koziris LP, Ratamess NA, Hakkinen K, Triplett-McBride NT, Fry AC, Gordon SE, Volek JS, French DN, Rubin MR, Gomez AL, Sharman MJ, Lynch JM, Izquierdo M, Newton RU, Fleck SJ (2002) Detraining produces minimal changes in physical performance and hormonal variables in recreationally strengthtrained men. Journal of Strength and Conditioning Research 16(3): 373-382.

Krotkiewski M, Gudmundsson P, Backstrom P, Mandroukas K (1982) Zinc and muscle strength and endurance. Acta Physiologica Scandinavia 116: 309.

Lukaski HC (2004) Vitamin and mineral status: Effects on physical performance. Nutrition 20: 632-644.

Petrie HJ, Stover EA, Horswill CA (2004) Nutritional concerns for the child and adolescent competitor. Nutrition 20: 620-631.

Richardson JH, Drake PD (1979) The effect of zinc on fatigue of striated muscle. Journal of Sports Medicine and Physical Fitness 19: 133.

Singh A, Moses FM, Deuster PA (1992) Chronic multivitamin-mineral supplementation does not enhance physical performance. Medicine and Science in Sports and Exercise 24(6): 726-732.

Telford RD, Catchpole EA, Deakin V, McLeay AC, Plank AW (1992) The effect of 7 to 8 months of vitamin/mineral supplementation on the vitamin and mineral status of athletes. International Journal of Sport Nutrition 2(2): 123-134.

Weight LM, Myburgh KH, Noakes TD (1988) Vitamin and mineral supplementation: Effect on the running performance of trained athletes. American Journal of Clinical Nutrition 47(2): 192-195. 
Copyright of Research in Sports Medicine is the property of Taylor \& Francis Ltd and its content may not be copied or emailed to multiple sites or posted to a listserv without the copyright holder's express written permission. However, users may print, download, or email articles for individual use. 